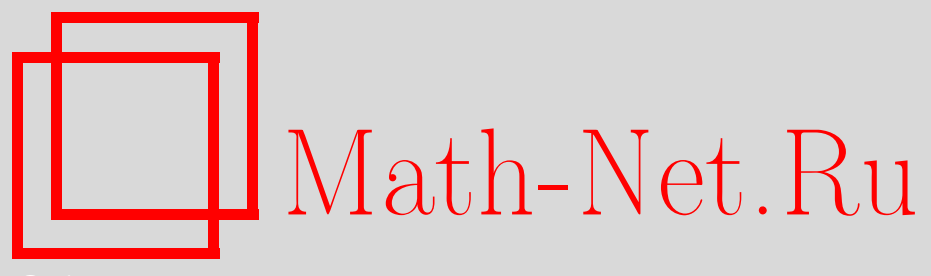

Е. В. Боровик, К. Ю. Федоровский, О связи неванлинновских и квадратурных областей, Матем. заметки, 2016, том 99, выпуск 3, 460-464

DOI: https://doi.org/10.4213/mzm11044

Использование Общероссийского математического портала Math-Net.Ru подразумевает, что вы прочитали и согласны с пользовательским соглашением http://www . mathnet.ru/rus/agreement

Параметры загрузки:

IP : 44.207 .124 .84

26 апреля 2023 г., 12:50:50

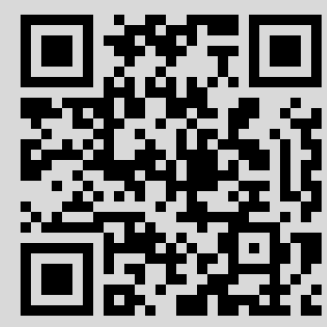




\section{О связи неванлинновских и квадратурных областей}

\section{Е. В. Боровик, К. Ю. Федоровский}

Пусть $\mathbb{D}=\{z \in \mathbb{C}:|z|<1\}$ - единичный круг в комплексной плоскости $\mathbb{C}$, а $\mathbb{T}=\{z \in \mathbb{C}:$ $|z|=1\}$ - единичная окружность. Для открытого множества $U \subset \mathbb{C}$ обозначим через $\operatorname{Hol}(U)$ пространство всех голоморфных, а через $H^{\infty}(U)$ пространство всех голоморфных ограниченных в $U$ функций.

ОПРедЕлЕниЕ 1. Ограниченная односвязная область $G \subset \mathbb{C}$ называется неванлинновской, если существуют функции $u, v \in H^{\infty}(G)$ такие, что равенство

$$
\bar{z}=\frac{u(z)}{v(z)}
$$

выполняется п.в. на $\partial G$ в смысле конформного отображения, т.е. для п.в. $\zeta \in \mathbb{T}$ имеет место равенство угловых граничных значений

$$
\overline{\varphi(\zeta)}=\frac{(u \circ \varphi)(\zeta)}{(v \circ \varphi)(\zeta)}
$$

где $\varphi$ - некоторое конформное отображение круга $\mathbb{D}$ на $G$.

Область $G$ называется локально неванлинновской, если существуют компакт $K \subset G$ и функции $u, v \in H^{\infty}(G \backslash K)$ такие, что равенство (1) выполняется п.в. на $\partial G$ в смысле конформного отображения.

Неванлинновские и локально неванлинновские области возникли в связи с задачами аппроксимации функций полианалитическими многочленами. Соответствующие определения были введены в [1; определение 3] и [2; определения 2.1 и 4.2] и [3; определение 2] (см. также [4], где приведен подробный обзор упомянутой аппроксимационной задачи). Класс всех неванлинновских областей будем обозначать через ND, а класс всех локально неванлинновских областей - через LND соответственно. Таким образом, ND $\subset$ LND.

Приведем несколько простых примеров. Пусть $D_{a, b}$ при $a>b>0$ - это область, ограниченная эллипсом

$$
\left\{z=x+i y: \frac{x^{2}}{a^{2}}+\frac{y^{2}}{b^{2}}=1\right\}
$$

и пусть

$$
D_{a, b}^{\prime}=\frac{1}{z}\left(D_{a, b}\right)
$$

(эта область называется овалом Нъюмана). Легко проверяется, что $\mathbb{D} \in \mathrm{ND}, D_{a, b} \in \mathrm{LND} \backslash$ $\mathrm{ND}$, a $D_{a, b}^{\prime} \in \mathrm{ND}$. Ряд свойств и примеров неванлинновских областей можно найти в работах [5] и [6]. В частности, отметим, что определение 1 корректно в том смысле, что оно не зависит от выбора конформного отображения $\varphi$. В силу граничной теоремы единственности Лузина-Привалова, отношение $u / v$ определено в области $G \in$ LND единственным образом. В случае, когда $G$ - это жорданова область со спрямляемой границей, равенство (1) можно понимать непосредственно как равенство угловых граничных значений почти всюду на $\partial G$. Кроме того, всегда можно считать, что функции $u$ и $v$ из определения 1 не имеют общих нулей.

Нам потребуется также понятие квадратурной области. Для $p \in[1, \infty]$ и для открытого множества $U \subset \mathbb{C}$ обозначим через $L_{a}^{p}(U)$ пространство Бергмана, состоящее из всех функций класса $L^{p}(U)$, голоморфных в $U$. Пространство Лебега $L^{p}(U)$ рассматривается относительно плоской меры Лебега $A(\cdot)$. Через $\langle T \mid f\rangle$ обозначается действие обобщенной функции (распределения) $T$ на функцию $f$, через $\operatorname{Supp} T$ - носитель $T$.

Работа выполнена при поддержке Российского научного фонда (грант № 14-21-00025).

DOI: $10.4213 / \mathrm{mzm} 11044$ 
ОПРедЕлЕниЕ 2. Область $G \subset \mathbb{C}$ называется квадратурной областью в широком смысле, если существует распределение $T$ с $\operatorname{Supp} T \subset G$ такое, что для любой функции $f \in$ $L_{a}^{1}(G)$ имеет место равенство

$$
\int_{G} f(z) d A(z)=\langle T \mid f\rangle .
$$

Область $G$ называется классической квадратурной областъю, если существуют конечное множество $\left\{a_{1}, \ldots, a_{N}\right\} \subset G$ и набор коэффициентов $c_{j, k}, j=1, \ldots, N, k=0, \ldots, n_{j}, N, n_{j} \in$ $\mathbb{Z}_{+}$, такие, что для любой функции $f \in L_{a}^{1}(G)$ выполнено равенство

$$
\int_{G} f(z) d A(z)=\sum_{j=1}^{N} \sum_{k=0}^{n_{j}} c_{j, k} f^{(k)}\left(a_{j}\right) .
$$

Равенства (2) и (3) называются квадратурными формулами (или квадратурными соотношениями) для $G$. Важно отметить, что они являются точными для всех голоморфных интегрируемых в области $G$ функций. Класс всех классический квадратурных областей обозначим через QD, а класс всех квадратурных областей в широком смысле через QDWS. Из теоремы о среднем вытекает, что $\mathbb{D} \in \mathrm{QD}$. Можно также показать, что $D_{a, b} \in \mathrm{QDWS} \backslash$ $\mathrm{QD}$, но $D_{a, b}^{\prime} \in \mathrm{QD}$. Квадратурные области естественно возникают во многих задачах комплексного анализа. Одно из первых упоминаний о квадратурных областях содержится в [7], а приведенные выше определения даны в том виде, как они содержатся в [8]. Отметим сборник [9], посвященный современному состоянию исследований квадратурных областей.

Оказывается, между неванлинновскими и квадратурными областями имеется интересная взаимосвязь, изучение которой и является целью настоящей заметки. Для того, чтобы увидеть эту связь нам потребуется понятие функции Шварца аналитической кривой. Пусть $B$ - жорданова область с аналитической границей $\Gamma$ (это означает, что простая замкнутая кривая $\Gamma$ является образом окружности $\mathbb{T}$ при отображении, однолистном в некоторой окрестности $\mathbb{T}$ ). Тогда существуют окрестность $U$ кривой $\Gamma$ и функция $S$, голоморфная в $U$, такие, что $\Gamma=\{z \in U: \bar{z}=S(z)\}$. Пусть $f$ - некоторое конформное отображение круга $\mathbb{D}$ на $G$. Тогда $f$ голоморфна и однолистна в круге $\{z \in \mathbb{C}:|z|<r\}$ при некотором $r>1$, а функция Шварца $S$ кривой $\Gamma$ связана с $f$ следующим образом:

$$
S(z)=\overline{f\left(\frac{1}{\overline{f^{-1}(z)}}\right) .}
$$

Таким образом, $B \in \mathrm{ND}$ в том и только том случае, когда функция Шварца $S$ ее границы мероморфна в $B$. Это же самое условие является необходимым и достаточным для того, чтобы $B \in \mathrm{QD}$ (см. [7; гл. XIV]). Отметим также, что условие мероморфности функции $S$ в $B$ эквивалентно тому, что $f$ - это рациональная функция, однолистная в $\mathbb{D}$ (см. $[7$; гл. XIV]). Итак, в случае жордановых областей с аналитическими границами классы ND и $\mathrm{QD}$ совпадают (и состоят из областей, которые являются образами единичного круга при отображении рациональными однолистными функциями). Выясним, на какой класс областей распространяется это наблюдение.

Теорема 1. В классе ограниченных односвязных областей в $\mathbb{C}$ имеют место следующие включения: QDWS $\subset \mathrm{LND}, \mathrm{QD} \subset \mathrm{ND}$, но $\mathrm{QD} \neq \mathrm{ND}$.

ДокАзАтЕльство. Пусть ограниченная односвязная область $G$ является квадратурной областью в широком смысле и пусть распределение $T$ взято из определения 2 для $G$. Докажем вначале, что существует функция

$$
S \in \operatorname{Hol}(G \backslash \operatorname{Supp} T) \cap C(\bar{G} \backslash \operatorname{Supp} T)
$$

такая, что $\bar{z}=S(z)$ на $\partial G$. При доказательстве этого факта мы будем использовать идеи и ряд конструкций из работ [10] и [8; гл. 4]. 
Пусть $I_{G}$ - характеристическая функция области $G$, т.е. $I_{G}(z)=1$ при $z \in G$ и $I_{G}(z)=0$ в противном случае. Рассмотрим преобразование Коши $F$ функции $I_{G}$, т.е.

$$
F(z)=\frac{1}{\pi} \int_{G} \frac{d A(\zeta)}{z-\zeta}
$$

Хорошо известно, что $F \in C(\mathbb{C})$. Кроме того, при $z \in \bar{G}$ имеет место равенство

$$
F(z)=\bar{z}+g(z)
$$

где $g \in C(\mathbb{C}) \cap \operatorname{Hol}(G)$. В самом деле, пусть $r>0$ таково, что круг $D=D(0, r)$ с центром в начале координат и радиусом $r$ содержит $\bar{G}$. Тогда

$$
F(z)=\frac{1}{\pi} \int_{D} \frac{d A(\zeta)}{z-\zeta}-\frac{1}{\pi} \int_{D \backslash G} \frac{d A(\zeta)}{z-\zeta},
$$

причем первый интеграл в этом равенстве равен $\bar{z}$, а второй представляет собой функцию, непрерывную всюду в $\mathbb{C}$ и голоморфную в $G$ и вне $\bar{D}$.

Рассмотрим теперь преобразование Коши $R$ распределения $T$ (т.е. $R=(\pi z)^{-1} * T$, где знак * обозначает свертку). Тогда $R$ - это голоморфная вне $\operatorname{Supp} T$ функция. Определим функцию $S:=-g-R$. Из отмеченных свойств функций $g$ и $R$ непосредственно вытекает, что

$$
S \in C(\bar{G} \backslash \operatorname{Supp} T) \cap \operatorname{Hol}(G \backslash \operatorname{Supp} T) .
$$

Для того, чтобы проверить равенство $\bar{z}=S(z)$ на $\partial G$ заметим, что из (2) вытекает, что $F(z)=-R(z)$ при $z \notin G$. Тогда для любого $z \in \partial G$ имеют место равенства

$$
S(z)=-g(z)-R(z)=\bar{z}-F(z)-R(z)=\bar{z} .
$$

Для того, чтобы показать, что $G \in \mathrm{LND}$ выберем некоторую односвязную окрестность $U$ множества $\operatorname{Supp} T$ такую, что $\bar{U} \subset G$. В этом случае мы оказываемся в условиях определения 1 при $u=S, v \equiv 1$ и $K=\bar{U}$.

Пусть теперь $G \in \mathrm{QD}$ является классической квадратурной. Тогда из (3) вытекает, что

$$
T=\sum_{j=1}^{N} \sum_{k=0}^{n_{j}} c_{j, k} \boldsymbol{\delta}_{a_{j}}^{(k)},
$$

где через $\boldsymbol{\delta}_{a}$ обозначена дельта-функция Дирака с носителем в точке $a \in \mathbb{C}$. Тогда

$$
R(z)=\frac{1}{\pi} \sum_{j=1}^{N} \sum_{k=0}^{n_{j}} \frac{k ! c_{j, k}}{\left(z-a_{j}\right)^{k+1}},
$$

т.е. $R$ является рациональной функцией. Но из этого следует, что функция $S=-g-R$ является мероморфной в $G$ откуда $G \in \mathrm{ND}$.

Покажем теперь, что QD $\neq$ ND для ограниченных односвязных областей общего вида. Пусть $G \in \mathrm{QD}$. Тогда существует мероморфная в $G$ и непрерывная в $\bar{G} \backslash\left\{a_{1}, \ldots, a_{N}\right\}$ функция $S$ такая, что $S(z)=\bar{z}$ на $\partial G$ (эту функцию называют односторонней функиией Швариа для $\partial G$ ). Из существования такой функции $S$ и из теоремы 5.2 работы [11] вытекает, что $\partial G$ состоит из конечного числа аналитических кривых, а из [6; теорема 2] или [12; теорема 1] вытекает, что существуют ND-области с нигде не аналитическими границами. Теорема доказана.

Отметим, что отношение $u / v$ из определения 1 для известных ND-областей с нигде не аналитическими границами не может быть выбрано непрерывным вплоть до $\partial G$. Это связано с тем, что в этих примерах полюсы соответствующего отношения накапливаются к точкам $\partial G$. В связи с этим наблюдением представляет интерес следующее утверждение. 
Теорема 2. Пусть $G$-жорданова область со спрямляемой границей. Предположим, что выполнены следующие условия:

(i) $G \in \mathrm{LND}$;

(ii) компакт $K$ и функиия $F=u / v$ взяты из определения 1 для $G$;

(iii) $\overline{Z(v)} \subset G$, где $Z(v)=\{z \in \mathbb{C}: v(z)=0\}$.

Тогда $G \in$ QDWS. Более того, если $G \in \mathrm{ND}$, то $G \in \mathrm{QD}$.

Условиям теоремы 2 удовлетворяют, например, области $D_{a, b}^{\prime} \in \mathrm{ND}$ и $D_{a, b} \in \mathrm{LND} \backslash \mathrm{ND}$.

Доказательство теоремы 2. Для компактного множества $Y \subset \mathbb{C}$ обозначим через $\widehat{Y}$ объединение $Y$ и всех ограниченных связных компонент множества $\mathbb{C} \backslash Y$. Пусть $\Gamma \subset$ $G \backslash \widehat{K}$ - это такая ломаная со сторонами, параллельными осям координат, что $K \subset \widehat{\Gamma}$ и $\overline{Z(v)} \subset \widehat{\Gamma}$. Таким образом, функция $F$ голоморфна на $Г$. Пусть $h$ - некоторая голоморфная в $\bar{G}$ функция. Тогда

$$
\int_{G} h(z) d A(z)=\frac{1}{2 i} \int_{\partial G} h(z) \bar{z} d z=\frac{1}{2 i} \int_{\partial G} h(z) F(z) d z=\frac{1}{2 i} \int_{\Gamma} h(z) \frac{u(z)}{v(z)} d z,
$$

причем в предпоследнем равенстве учтено, что равенство (1) в рассматриваемом случае может пониматься как равенство угловых граничных значений почти всюду на $\partial G$. Определим распределение $T$ с носителем на $\Gamma$ следующим образом:

$$
\langle T \mid \varphi\rangle:=\frac{1}{2 i} \int_{\Gamma} \varphi(z) \frac{u(z)}{v(z)} d z, \quad \varphi \in C_{0}^{\infty}(\Omega),
$$

где $\Omega$ - некоторая ограниченная односвязная область, содержащая $\bar{G}$. Тогда для любой функции $h$, голоморфной в $\bar{G}$, имеет место равенство

$$
\int_{G} h(z) d A(z)=\langle T \mid h\rangle .
$$

Покажем, что это равенство выполнено и для любой функции $g \in L_{a}^{1}(G)$. Учитывая то, что пространство $H^{\infty}(G)$ плотно в $L_{a}^{1}(G)$, и применяя теорему Фаррела-Рубеля-Шилдса (см. теорему 5.1 в [13; гл. VI]), найдем последовательность многочленов $\left\{p_{m}\right\}_{m=1}^{\infty}$ такую, что $\left|p_{m}(z)\right| \leqslant|g(z)|$ и $p_{m}(z) \rightarrow g(z)$ при $m \rightarrow \infty$ для любой точки $z \in G$. Тогда

$$
\begin{aligned}
\int_{G} g(z) d A(z) & =\lim _{m \rightarrow \infty} \int_{G} p_{m}(z) d A(z)=\lim _{m \rightarrow \infty}\left\langle T \mid p_{m}\right\rangle \\
& =\lim _{m \rightarrow \infty} \frac{1}{2 i} \int_{\Gamma} p_{m}(z) \frac{u(z)}{v(z)} d z=\frac{1}{2 i} \int_{\Gamma} g(z) \frac{u(z)}{v(z)} d z=\langle T \mid g\rangle,
\end{aligned}
$$

откуда вытекает, что (4) выполняется для любой функции класса $L_{a}^{1}(G)$. Таким образом, область $G$ является квадратурной областью в широком смысле.

Если $G$ - это неванлинновская область, то $K=\varnothing$ и для любой функции $g$, голоморфной в области $G$, интеграл

$$
\int_{\Gamma} g(z) \frac{u(z)}{v(z)} d z
$$

можно вычислить, используя теорему Коши о вычетах. Несложно проверить, что этот интеграл будет равен сумме значений функции $g$ и ее производных в точках множества $Z(v)$. Другими словами, это означает, что квадратурное соотношение (4) в рассматриваемом случае будет классическим квадратурным соотношением, а область $G$ - классической квадратурной областью. Теорема доказана.

Теоремы 1 и 2 устанавливают связь между неванлинновскими и квадратурными областями в классе ограниченных односвязных областей в $\mathbb{C}$. В связи с этим отметим, что определение квадратурной области не предполагает ни ограниченности, ни односвязности. Более того, в [14] показано, что существуют многосвязные квадратурные области. 


\section{СПИСОК ЦИТИРОВАННОЙ ЛИТЕРАТУРЫ}

[1] К. Ю. Федоровский, Матем. заметки, 59:4 (1996), 604-610. [2] Д. Д. Кармона, П. В. Парамонов, К. Ю. Федоровский, Матем. сб., 193:10 (2002), 75-98. [3] J. J. Carmona, K. Yu. Fedorovskiy, Selected Topics in Complex Analysis, Oper. Theory Adv. Appl., 158, Birkhäuser Verlag, Basel, 2005, 109-130. [4] М. Я. Мазалов, П. В. Парамонов, К. Ю. Федоровский, УМН, 67:6 (2012), 53-100. [5] К. Ю. Федоровский, Комплексный анализ и приложения, Тр. МИАН, 253, Наука, М., 2006, 204-213. [6] А. Д. Баранов, К. Ю. Федоровский, Матем. сб., 202:12 (2011), 3-22. [7] P. Davis, The Schwarz Function and Its Applications, Carus Math. Monogr., 17, Math. Assoc. Amer., Buffalo, NY, 1974. [8] H. S. Shapiro, The Schwarz Function and Its Generalization to Higher Dimensions, Univ. Arkansas Lecture Notes Math. Sci., 9, John Wiley and Sons, New York, 1992. [9] Quadrature Domains and Their Applications, Oper. Theory Adv. Appl., 156, Birkhäuser Verlag, Basel, 2005. [10] D. Aharonov, H. S. Shapiro, J. Analyse Math., 30 (1976), 39-73. [11] M. Sakai, Acta Math., 166:3-4 (1991), 263-297. [12] М. Я. Мазалов, Матем. заметки, 62:4 (1997), 629-632. [13] Т. Гамелин, Равномерные алгебры, Мир, М., 1973. [14] D. Crowdy, J. Marshall, SIAM J. Appl. Math., 64:4 (2004), 1334-1359.

\section{Е. В. Боровик}

Московский государственный технический 11.10 .2015 университет имени Н. Э. Баумана;

Институт прикладной математики им. М. В. Келдыша РАН, г. Москва

E-mail: katrina_borovik@mail.ru

\section{К. Ю. Федоровский}

Московский государственный технический университет имени Н. Э. Баумана; Институт прикладной математики им. М. В. Келдыша РАН, г. Москва

E-mail: kfedorovs@yandex.ru 